\title{
Variabilité climatique et production maraîchère dans la plaine inondable d'Ahomey-Gblon au Bénin
}

\author{
Séraphin Capo ATIDEGLA ${ }^{1 *}$, Hervé Dègla KOUMASSI ${ }^{2}$ et Elvire Sènan HOUSSOU ${ }^{1}$ \\ ${ }^{I}$ Faculté des Sciences Agronomiques, Université d'Abomey-Calavi, Cotonou, Bénin. \\ ${ }^{2}$ Faculté des Sciences Humaines et Sociales (FASHS), Université d'Abomey-Calavi, Cotonou, Bénin. \\ *Auteur correspondant, E-mail : atideglaser@gmail.com
}

\section{RESUME}

Le maraîchage pratiqué dans les plaines inondables fait partie des activités qui assurent la diversification des habitudes alimentaires dans le but de contribuer à la sécurité alimentaire et à la réduction de la pauvreté. Cependant, dans les pays au Sud du Sahara, la variabilité climatique représente une grande menace pour la croissance et le développement durable. La présente étude se propose d'évaluer les effets de la variabilité climatique sur la production maraîchère dans la plaine inondable d'Ahomey-Gblon. Les données utilisées sont issues entre autres de l'analyse de la variabilité des facteurs climatiques, des tests statistiques, des investigations socio-anthropologiques et des tests de rendement de trois principales cultures. Les résultats de cette étude révèlent que les conséquences des perturbations climatiques sur le maraîchage se traduisent entre autres par les inondations, les sécheresses, la baisse des rendements agricoles et le bouleversement du calendrier agricole classique. Par exemple, au cours des trois dernières campagnes (2014, 2015 et 2016), les rendements de la tomate, du piment et de la patate douce ont régressé respectivement de $31 \%, 34 \%$ et $17 \%$. Pour surmonter les effets des variabilités climatiques, les producteurs ont développé des stratégies d'adaptation telles que l'utilisation de semences à cycle court, l'agriculture de contre saison, la modification de la date de semis, la diversification des sources de revenus, la diversification agricole et l'irrigation des parcelles et des cultures. Il urge donc d'accompagner les producteurs dans le renforcement de leurs capacités d'adaptation.

(C) 2017 International Formulae Group. All rights reserved.

Mots clés : Baisse de rendements, stratégies d'adaptation, agriculture de contre saison, irrigation,

\section{Climate variability and vegetables production in the liable to flooding plain of Ahomey-Gblon in Benin}

\begin{abstract}
Vegetables production in the liable to flooding plains counts among the activities which ensure the diversification of human feeding in order to contribute to food security and poverty reduction. However, in the South of Sahara countries, climatic variability represented a big constraint for an economic growth and a sustainable development. The present study aims to evaluate the effects of climate variability on vegetable production in the liable to flooding plain of Ahomey-Gblon. The data used come among others from the analysis of climate factors, the statistic tests, the social and anthropologic investigations and the yield trials of three main crops. The results of the study revealed that climatic disturbances on vegetables production leaded up to flood decline of yields and the disruption of traditional agricultural calendar. For example, during the three last production campaigns (2014, 2015 and 2016), the yields of tomato, pepper and sweet potato decrease respectively of $31 \%, 34 \%$ and $17 \%$. To overcome the effects of climate variability, producers developed
\end{abstract}


adaptation strategies such as the use of early varieties seeds, the dry season cropping, the diversification income sources, of income, the agricultural diversification, the variation of sowing dates and the irrigation of plots and crops. Therefore, it is urgent to support the farmers in the reinforcement of their adaptation capacities.

(C) 2017 International Formulae Group. All rights reserved.

Keywords: Decline of yields, adaptation strategies, dry season cropping, irrigation.

\section{INTRODUCTION}

La variabilité climatique est l'un des phénomènes les plus importants de notre époque. En Afrique, elle représente une grande menace pour la croissance et le développement durable, ainsi que pour l'atteinte des Objectifs du Millénaire pour le Développement (OMD). En effet, selon GIEC (2007), les communautés pauvres seront les plus vulnérables du fait de leurs capacités d'adaptation limitées et leur grande dépendance de ressources à forte sensitivité climatique telles que les ressources en eau et les systèmes de production agricole. En cas d'échec, les $40 \%$ de la population mondiale la plus pauvre, soit environ 2,6 milliards de personnes, seront condamnées à un futur comportant moins d'opportunités (PNUD, 2007). Dans la plupart des pays et régions de ce continent, on s'attend à ce que la production agricole et l'accès à la nourriture soient sérieusement compromis par la variabilité du climat. $\mathrm{Au}$ Bénin où l'agriculture constitue la base de l'économie avec une contribution de $36 \%$ au Produit Intérieur Brut et de $88 \%$ aux recettes d'exportation (Houssou, 2014), les impacts négatifs du changement climatique ne sont plus à démontrer. Du moment où, l'agriculture béninoise reste essentiellement pluviale, les paramètres agro-climatiques présentent des particularités contraignantes pour l'agriculture et la foresterie surtout dans le Sud et l'Extrême Nord qui connaissent parfois de graves sécheresses (MEPN, 2008). Les travaux de Ogouwalé (2006), Vissin (2007) et Koumassi (2014) ont montré que les climats du Bénin sont caractérisés par une variabilité pluviométrique, une réduction de la durée de la saison agricole et une hausse des températures minimales. De ce fait, l'agriculture subit de plein fouet, les contrecoups de la variabilité climatique, notamment celle des précipitations et de la température. Par conséquent, le secteur agricole se trouve confronté à des baisses de rendement de plus en plus remarquables $\mathrm{du}$ fait des pratiques culturales utilisées (GIEC, 2007). Cette situation conduit les paysans vers les terres non encore cultivées telles que les bas-fonds et les plaines inondables très fertiles qui offrent des possibilités agricoles et piscicoles. Ces zones représentent des atouts incontestables pour la production agricole (Sossa, 1996). Ainsi, l'imminence des mesures d'adaptation pour les $61,1 \%$ de la population du pays qui vivent en milieu rural (INSAE, 2003) et dépendant de l'agriculture pour leur subsistance se justifie à plus d'un titre.

En effet, selon UICN (2000), les zones humides font partie des écosystèmes les plus productifs de la terre; elles sont tantôt décrites comme les «reins du paysage» pour les fonctions qu'elles remplissent dans les cycles hydrologique et chimique et tantôt comme des «supermarchés biologiques» en raison des vastes chaînes alimentaires et de la riche diversité biologique qu'elles entretiennent. Les bas-fonds et les plaines inondables font de plus en plus l'objet d'une exploitation croissante. La qualité chimique des sols est bonne et l'eau disponible. Elles représentent une alternative économique intéressante pour les paysans béninois qui peuvent y développer une production de sécurité ou de rente (sorgho, maïs, riz) en saison des pluies. En saison sèche, ce sont des zones de pâturage, qui offrent aussi d'intéressantes possibilités de maraîchage, d'arboriculture et de cultures de contre-saison. Pour Atidégla (2012), les plaines inondables et les bas-fonds bénéficient des dépôts d'éléments provenant de l'érosion des versants par les pluies et présentent des sols généralement plus profonds, plus riches et plus humides que ceux du plateau. 
Le maraîchage pratiqué dans les basfonds et les plaines inondables fait partie des activités qui assurent la diversification des habitudes alimentaires dans le but de contribuer à la sécurité alimentaire et à la réduction de la pauvreté. Cependant, la productivité maraîchère est aujourd'hui menacée par les fluctuations persistantes de certains paramètres climatiques que sont notamment : la température, les précipitations, le vent, l'ensoleillement. Dès lors, l'imminence des mesures d'adaptation pour les $61,1 \%$ de la population du pays qui vivent en milieu rural (INSAE, 2003) et dépendant de l'agriculture pour leur subsistance se justifie à plus d'un titre. C'est dans l'optique d'appréhender l'effet de cette variabilité climatique sur la production maraîchère et les stratégies d'adaptation des producteurs de la plaine inondable d'Ahomey-Gblon (Commune de Sô-Ava) que la présente étude a été réalisée.

\section{MATERIEL ET METHODES Zone d'étude}

La commune de Sô-Ava d'une superficie de $218 \mathrm{~km}^{2}$ est comprise entre $6^{\circ} 24^{\prime}$ et $6^{\circ} 38^{\prime}$ de latitude Nord et $2^{\circ} 21^{\prime}$ et $2^{\circ} 30^{\prime}$ de longitude Est. Située dans le département de l'Atlantique, elle occupe une partie de la basse vallée du fleuve Ouémé et de la rivière Sô à laquelle elle doit sa toponymie (Kouhoundji 2011) tandis que le village d'étude appelé Ahomey-Gblon est localisé dans l'arrondissement de Sô-Ava. Située à l'Ouest du fleuve Ouémé, Sô-Ava est une commune lacustre, distante de $40 \mathrm{~km}$ de Cotonou, la capitale économique dont une partie occupe la plaine d'inondation de la rivière Sô. Comme toute la région méridionale, Sô-Ava jouit d'un climat de type sub-équatorial caractérisé par deux saisons de pluie qui sont alternées par deux saisons sèches à durées inégales se répartissant comme suit: une grande saison pluvieuse de avril à juillet et une petite saison sèche de juillet à août, une petite saison pluvieuse de septembre à octobre et une grande saison sèche de novembre à mars se confondant à la décrue. La moyenne pluviométrique annuelle est de $1300 \mathrm{~mm}$ et la température moyenne annuelle est de $27,2^{\circ} \mathrm{C}$.
La frontière entre les Communes de Sô-Ava et d'Abomey-Calavi est la rivière Sô. D'une longueur de $84,4 \mathrm{~km}$, la rivière Sô fait partie des défluents du fleuve Ouémé (Mairie Abomey-Calavi, 2012). Ses plus forts débits sont observés pendant les crues de l'Ouémé qui s'installent entre le mois d'août et de novembre.

$\mathrm{La}$ végétation est verdoyante. On trouve dans les dans les plaines et bas-fonds des essences telles que le Paspalumvaginatum et le Typha australis (herbes des marécages). Depuis bientôt 20 ans, la Jacinthe d'eau (Eichorniacrassipes) et la salade d'eau douce (Pistiastratiotes) envahissent les cours et plans d'eau. Des îlots de belles forêts couvrent les berges des villages comme HouédoGbadji, Dékanmè, Gbessou et AhomeyLokpo. Enfin, les cocotiers (Cocos nucifera) et les palmiers à huile (Elaeis guineensis) complètent ce paysage de rêve.

\section{Données climatologiques Les données climatologiques (précipitations, températures, évapotranspiration, vents) utilisées pour caractériser la variabilité climatique dans la commune de Sô-Ava ont été extraites des fichiers de l'Agence pour la Sécurité de la Navigation (ASECNA) Cotonou. La station météorologique d'Allada a fourni les données permettant d'étudier la répartition spatio- temporelle des précipitations. Grâce à toutes ces données, l'interprétation de la perception de l'évolution du climat a été faite sur la période 1970-2016 soit au moins quatre (4) décennies.}

\section{Traitement des données climatologiques}

Le régime thermo-pluviométrique a été fait à partir de l'analyse des indices pluviométriques sur la série 1970 à 2015. La moyenne arithmétique est employée pour étudier les régimes pluviométriques et thermométriques sur la station de Cotonou. Elle est le paramètre fondamental de tendance centrale. Elle s'exprime de la façon suivante :

$$
\bar{X}=\frac{1}{n} \sum_{i=1}^{n} x i
$$

La moyenne $\bar{X}$ nous a permis de caractériser l'état pluvio-thermométrique 
moyen et de mettre au point quelques indices de dispersion. Par ailleurs, l'étude des tendances pluviométriques et hydrologiques générales a été faite à partir du calcul des moyennes mobiles et de la régression. Les moyennes mobiles sont centrées sur cinq (5) ans. Cette méthode a permis d'avoir des séries pluviométriques thermométriques lissées sur la station au pas de temps journalier, mensuel et annuel. Enfin, la détection de rupture de stationnarité dans les séries pluviométriques et thermométriques a été faite à l'aide du test de Pettitt (1979) et celui de Buishand (1984). En effet, une «rupture» se définit par un changement dans la loi de probabilité des variables aléatoires dont les réalisations successives définissent les séries chronologiques étudiées (Lubes-Niel, 1994).

\section{Détermination de la durée des saisons culturales et couverture des besoins en eau des cultures}

La saison culturale (SC) ou saison de pluie potentiellement utile est définie comme la suite des décades consécutives pour lesquelles la valeur de la capacité d'évapotranspiration d'un couvert végétal (CEcv en $\mathrm{mm} /$ jours) est significativement supérieure ou égale à $50 \%$ de l'évapotranspiration de référence (ETP) de la période considérée (Eldin, 1989; Morel, 2004 ; Noufe et al., 2011 ; Noufe et al., 2015). $\mathrm{Ce}$ seuil est à rapprocher des valeurs du rapport ETM/ETP considérées en début et fin de cycle de culture, et utilisées en tant qu'indices de satisfaction des besoins en eau des cultures en conditions naturelles non irriguées ; ETM est considérée ici comme l'évapotranspiration réelle maximale d'une culture donnée (Noufe et al., 2015). Ainsi, le besoin en eau optimum d'une culture donnée ou l'évapotranspiration maximale (ETM) s'obtient par le produit de l'ETP et du coefficient cultural $(\mathrm{kc})$ de cette culture ; ce qui revient algébriquement à $\mathrm{ETM}=\mathrm{Kc} \mathrm{x}$ ETP. Cette valeur a été divisée en trois phases : pré-humide, humide, et post-humide.

A partir de ces formules, la pluie $(\mathrm{P})$ a été alors comparée à l'évapotranspiration potentielle (ETP) mensuelle ce qui permet de caractériser les saisons culturales.
Selon Noufe et al. (2015), pour un mois donné, si les pluies (apport d'eau) sont supérieures à l'ETP (la demande en eau), cela voudra dire que les cultures ne manqueront pas d'eau. De la même manière, le rapport ETP/2 représente un besoin hydrique moyen qui permet à un couvert végétal de démarrer son cycle végétatif et de le terminer dans les conditions optimales. En fin de compte, le bilan climatique calculé traduit le rythme des excédents ou des déficits en eau à l'échelle mensuelle ; car il exprime la différence entre les totaux pluviométriques $(\mathrm{P})$ et la valeur de l'évapotranspiration (ETP), constituant soit le déficit, soit l'excès d'eau disponible pour la recharge de la nappe et pour l'écoulement (Cocheme et Franquin, 1969, cités par Noufe et al., 2015). D'après ces auteurs, la formule du bilan climatique (BC) est la suivante :

$\mathrm{BC}=\mathrm{P}-\mathrm{ETP}$

Avec : $\mathrm{BC}=$ bilan climatique $(\mathrm{mm}) ; \mathrm{P}=$ pluviométrie totale mensuelle $(\mathrm{mm})$; ETP : évapotranspiration potentielle mensuelle (mm).

Ainsi, si :

$\mathrm{P}-\mathrm{ETP}<0, \mathrm{BC}$ est déficitaire ;

$\mathrm{P}-\mathrm{ETP}>0, \mathrm{BC}$ est excédentaire ;

$\mathrm{P}-\mathrm{ETP}=0, \mathrm{BC}$ est équilibré.

Sur la base des données climatologiques obtenues à l'ASECNA de Cotonou, la courbe ombro-thermique combinée à la courbe de la vitesse moyenne mensuelle du vent de la Commune de Sô-Ava 2014-2016 ont été réalisées afin d'apprécier la relation entre le vent, la température et la pluie. Il en est de même du diagramme climatique de Franklin (1968) que nous avons appliqué à la zone d'étude afin de caractériser les saisons culturales. Enfin sur la base de la formule $\mathrm{BC}=\mathrm{P}-\mathrm{ETP}$, le bilan climatique a été fait pour mettre en exergue les mois déficitaires et ceux déficitaires en matière de disponibilité d'eau.

\section{Evaluation des rendements}

Deux saisons des pluies (la grande et la petite) ont été offertes à l'agriculture dans la plaine inondable d'Ahomey-Gblon où les principales cultures pratiquées par la plupart des producteurs sont : la tomate, le piment, la patate douce, le gombo et les légumesfeuilles. Pour le test, il s'est agi d'apprécier 
les rendements de la tomate, du piment et la patate douce sur les trois dernières années (2014, 2015 et 2016). Pour les trois cultures, les variétés locales de cycle court ont été utilisées (100 à 110 jours pour la tomate et le piment et 120 jours pour la patate douce) et elles ont été installées dès les décades qui ont suivi le démarrage des saisons culturales. En effet, les dates d'installation des pluies et la durée des saisons humides sont essentielles en agriculture pluviale (Balme, 2004); car elles déterminent les dates de semis et les périodes pendant lesquelles les cultures bénéficient d'une quantité d'eau suffisante pour leur développement.

Trois paysans ont été suivis par spéculation au cours des trois campagnes agricoles pour la grande saison de pluie d'avril à juillet. Il s'agit des paysans $\mathrm{A}, \mathrm{B}$ et $\mathrm{C}$ pour respectivement la tomate, le piment et la patate douce. La parcelle d'essai retenue par paysan et par campagne est de $400 \mathrm{~m}^{2}$. Les divers entretiens (sarclage et protection phytosanitaire) ont été identiques au niveau de chaque parcelle. Les producteurs de la zone n'utilisent pas les engrais minéraux du fait du niveau élevé de fertilité de la zone de production. Le rendement étant la quantité de produit récoltée à l'hectare, il est procédé au moment de la récolte à la fin de chaque campagne, à la pesée des produits obtenus au niveau du carré de rendement installé sur chacune des six parcelles. La balance de portée $5 \mathrm{~kg}$ a été utilisée pour l'opération. Cette opération consiste à délimiter sur chaque parcelle, $1 \mathrm{~m}^{2}$ de parcelle où la densité des plants est la plus forte possible. A la fin du cycle, ces carrés de densité deviennent les carrés de rendement au moment de la récolte et les produits issus de ces carrés sont systématiquement pesés.

\section{Traitement des données relatives aux} rendements agricoles

Les données recueillies pour les rendements agricoles ont été traitées à l'ordinateur au moyen du logiciel Excel. Spécifiquement, les moyennes par spéculation et par année $(2014,2015$ et 2016) ont été calculées puis comparées et les résultats mettant en exergue l'évolution (négative ou positive) ont été illustrés par un tableau.

\section{RESULTATS}

Variabilité pluviométrique

La Figure 1 montre les valeurs d'indices pluviométriques interannuels (variables pluviométriques annuelles, centrées et réduites) à la Station de Cotonou de 1970 2016.

L'analyse de cette figure montre bien la forte variabilité interannuelle des hauteurs de pluie et le contexte de déficit des pluies annuelles enregistrées par l'ASECNA.

L'analyse a permis d'observer une instabilité de l'évolution pluviométrique de 1970 à 2015. Les déficits les plus prononcés sont enregistrés au cours des années 1971, 1973, 1974, 1976, 1980, 1984 et ceux excédentaires sont 1979, 1987, 1988, 1993, 1997, 2009, 2010. Cela a permis d'identifier trois phases dans l'évolution de la pluviométrie sur la station. La première phase est marquée par la prédominance des déficits sur la station, et concerne la période 19701986. La deuxième sous-série est caractérisée par des excédents pluviométriques entre la période 1987-2005. La troisième phase est caractérisée par une très forte instabilité dans l'évolution de la pluviométrie et concerne la période 2005- 2016.

Pour mieux apprécier ces différentes sous périodes, la rupture de stationnarité dans les épisodes pluvieux ont été déterminés.

\section{Détection de rupture de stationnarité dans la série pluviométrique}

Les Figures 2 et 3 traduisent respectivement les tests de Pettitt et de Buishand et ellipse de bois appliqués aux séries pluviométriques annuelles (1970-2016).

Le test de Pettitt et le test de Buishand appliqués aux séries pluviométriques montrent une rupture de stationnarité significative au seuil de 95\% au début des années 1986. Il apparaît donc deux grandes sous périodes notamment la période sèche de 1970 à 1986 et la période d'excédent pluviométrique de 1986 à 2015 .

\section{Tendances thermométriques}

La Figure 4 montre la variation de l'indice thermométrique à la station Cotonou aéroport. 
De l'analyse de cette figure, il ressort qu'une tendance générale à la hausse s'observe aussi dans l'évolution des températures maximales et minimales sur la période d'étude. Les températures minimales et maximales ont enregistré les plus fortes hausses respectives de $+1,52$ à $+1,72{ }^{\circ} \mathrm{C}$ en 1970, 19711973 dans les températures minimales et 0,52 à 0,91 en 1973,1983 et 2010 dans les températures maximales. Le record d'anomalie de la température moyenne est atteint en 1998 , avec $+2,4^{\circ} \mathrm{C}$.

\section{Début de la saison des pluies}

La Figure 5 présente l'évolution les dates de démarrage des saisons de pluie sur la période de 1970 -2015.

L'analyse de cette figure révèle une variation dans la date de démarrage de la grande saison des pluies. Sur l'ensemble de la période considérée, on note un retard dans le démarrage de la grande saison des pluies. En effet, la date de démarrage de la grande saison des pluies dans le secteur d'étude est le 15 mars. Mais on note une forte variation de cette date sur l'ensemble de la période avec une tendance générale à la normale. Les retards les plus significatifs obtenus dépassent parfois 60 jours comme le cas des années 1970, 2000 et 2011 où la saison agricole a démarré respectivement le 17, 18, 21 mai.

\section{Raccourcissement des saisons de pluies}

La Figure 6 présente l'évolution interannuelle des longueurs des saisons pluvieuses dans le secteur d'étude.

L'analyse de cette figure indique un raccourcissement des saisons pluvieuses dans le secteur d'étude. En effet, on note une tendance générale à la baisse du nombre de jours de pluie au cours des saisons pluvieuses. Les saisons pluvieuses deviennent de plus en plus courtes dans le secteur d'étude au regard du nombre de jours de pluies.

Impact de la variation des paramètres climatiques sur la production maraîchère au cours des années 2014, 2015 et 2016

Dans la zone d'étude, les paramètres climatiques les plus déterminants ayant une action directe sur les cultures et leurs besoins d'irrigation sont entre autres : les précipitations, la température, le vent et l'évapotranspiration (ETP).

Situation climatique et couverture des besoins en eau des cultures

Le volume d'eau nécessaire à la production végétale dépend des conditions du sol, de la variété des cultures, du vent et des températures. Une élévation de la température moyenne (29-30 $\left.{ }^{\circ} \mathrm{C}\right)$ accompagnée d'un accroissement de la vitesse du vent pendant la saison des pluies n'est pas bénéfique pour les cultures. La Figure 7 montre la relation entre le vent, la température et la pluie.

Il ressort de la Figure 7 que le trimestre sec allant de Janvier à mars a connu un accroissement de la vitesse du vent. En outre, la grande saison des pluies (avril-juillet) est plus ventilée que la petite (septembre octobre).

L'application de la méthode d'analyse agro climatique (Figure 8 ) en région tropicale de Franklin a donné les résultats suivants :

de fin novembre à janvier, la hauteur de pluie $\mathrm{P}$ est inférieure à ETP/2. Cette période incluse dans la saison de décrue ou contre saison ou grande saison sèche (novembre-avril) correspond à la préparation du sol et à l'installation des cultures. Au cours de cette période, les besoins en eau de la plante ne peuvent être satisfaits par l'eau de pluie mais par la réserve d'eau disponible dans le sol ou par une irrigation d'appoint ;

de février à fin juillet et de fin août à novembre, nous avons $\mathrm{P}$ supérieur à l'ETP. Ces mois correspondent à une période humide où les besoins en eau de la plante peuvent être satisfaits pleinement ou tout au moins globalement ;

il n'y a aucune période au cours de laquelle, la hauteur de pluie $\mathrm{P}$ inférieure à l'ETP. Ceci traduit le fait qu'il n'y a aucun moment où on assiste à un épuisement total du stock emmagasiné au cours de la période précédente.

Toutefois en liaison avec la Figure 7, il convient de souligner que l'élévation de la vitesse $\mathrm{du}$ vent combinée à la forte température a engendré la hausse de l'ETP au cours du trimestre allant de janvier à mars.

La Figure 9 traduit au mieux l'évolution du bilan climatique (P-ETP) moyen mensuel de 
2014 à 2016 dans la Commune. C'est un indicateur de disponibilité en eau du sol. Il traduit le rythme des excédents ou des déficits en eau. En effet, de l'analyse de cette figure, il ressort que :

- $\quad$ les déficits en eau ont été enregistrés dans l'ordre croissant au cours des mois d'août, novembre, février, janvier et décembre avec respectivement $-3,34 \mathrm{~mm},-8,2 \mathrm{~mm}$, $12,9 \mathrm{~mm}, \quad-48,07 \mathrm{~mm}$ et $-80,02 \mathrm{~mm}$. Ces résultats posent le problème de la non disponibilité de la réserve d'eau pendant les quatre premiers mois de la grande saison sèche consacrés à l'agriculture de contre saison ;

- pendant les sept autres mois de l'année, la hauteur des précipitations dépasse l'ETP, il n'y a pas de déficit pluviométrique. Les mois de mai, juin et octobre sont les plus pluvieux avec respectivement (P-ETP) égale à $91,73 \mathrm{~mm}, 260,26 \mathrm{~mm}$ et $122,57 \mathrm{~mm}$. C'est n'est seulement qu'au cours de ces mois qu'un surplus d'eau susceptible de recharger les retenues d'eau et la nappe phréatique est observé ; la valeur $(-3,34 \mathrm{~mm})$ du déficit climatique en août montre bien que ce mois correspond à la petite saison sèche.

Rendements de la tomate, du piment et de la patate douce au cours des années 2014, 2015 et 2016

L'évolution des rendements moyens qui se dégagent des essais des principales cultures (tomate, piment et patate douce) au cours des années 2014, 2015 et 2016 est illustrée par le Tableau 1.

De l'analyse de ce tableau, il ressort que les rendements moyens obtenus au fil des trois années de production de la grande saison (avril-juillet) sur la plaine ont connu de régression d'année en année pour les trois cultures. De 2014 à 2016, la tomate est passée de $19,25 \mathrm{t} /$ ha à $12 \mathrm{t} /$ ha en 2016 soit une régression de $38 \%$, le piment de $7 \mathrm{t} / \mathrm{ha}$ à $4 \mathrm{t} / \mathrm{ha}$ avec une régression de $43 \%$ et la patate douce de $10,5 \mathrm{t} /$ ha à $8,2 \mathrm{t} /$ ha soit un taux de régression de $22 \%$. Il faut tout de même signaler que ces cultures n'ont bénéficié d'aucun apport d'engrais minéral ou organique.

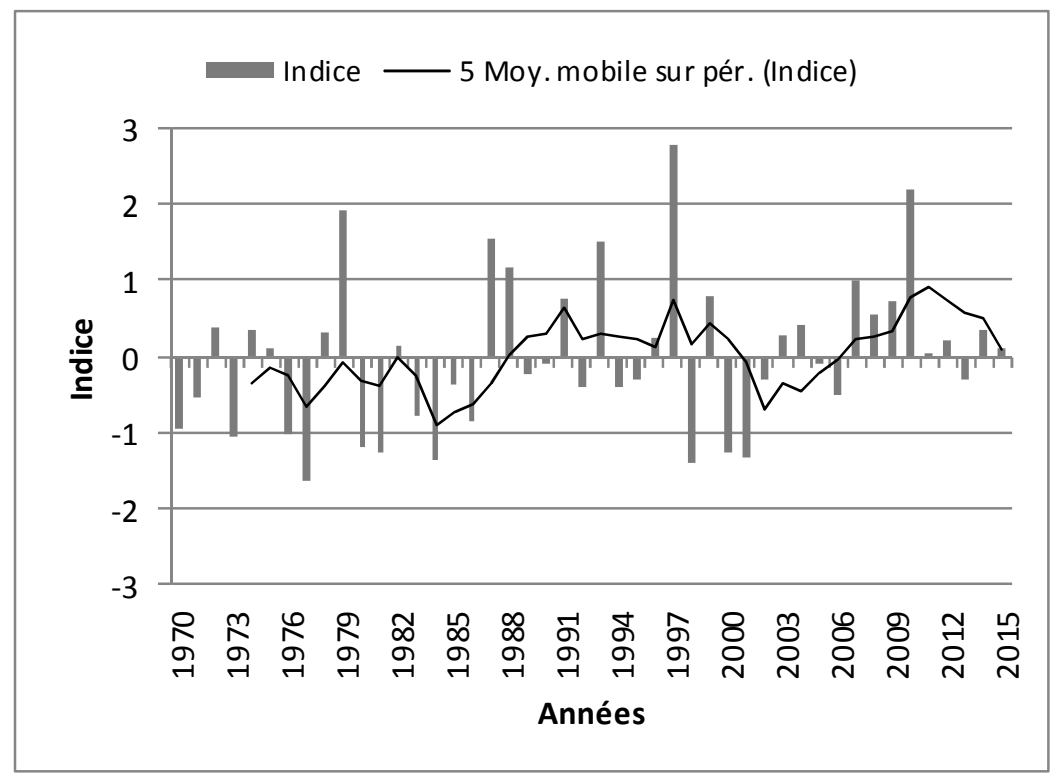

Figure 1 : Indice pluviométrique à Sô-Ava. 


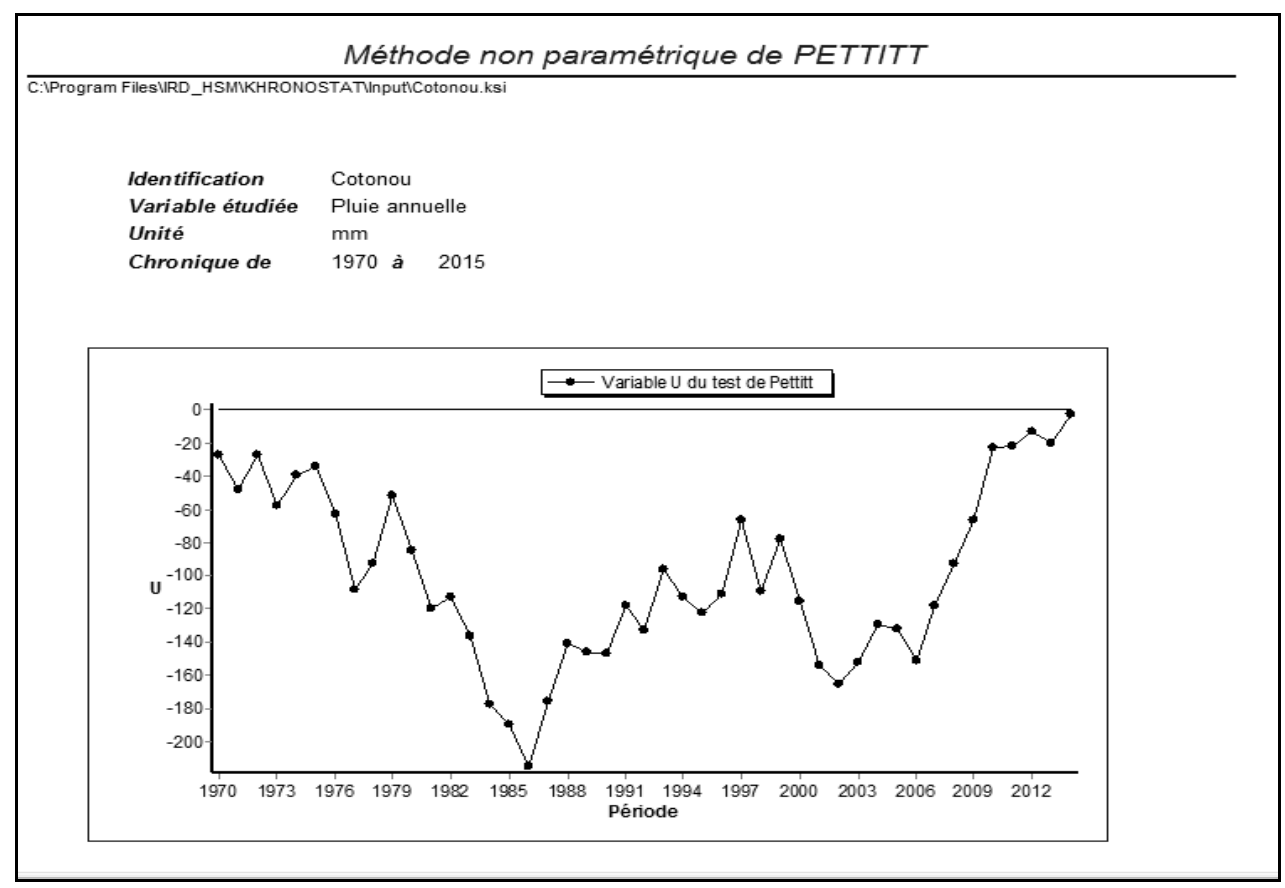

Figure 2 : Rupture de stationnarité par le test de Pettitt à la station de Cotonou.

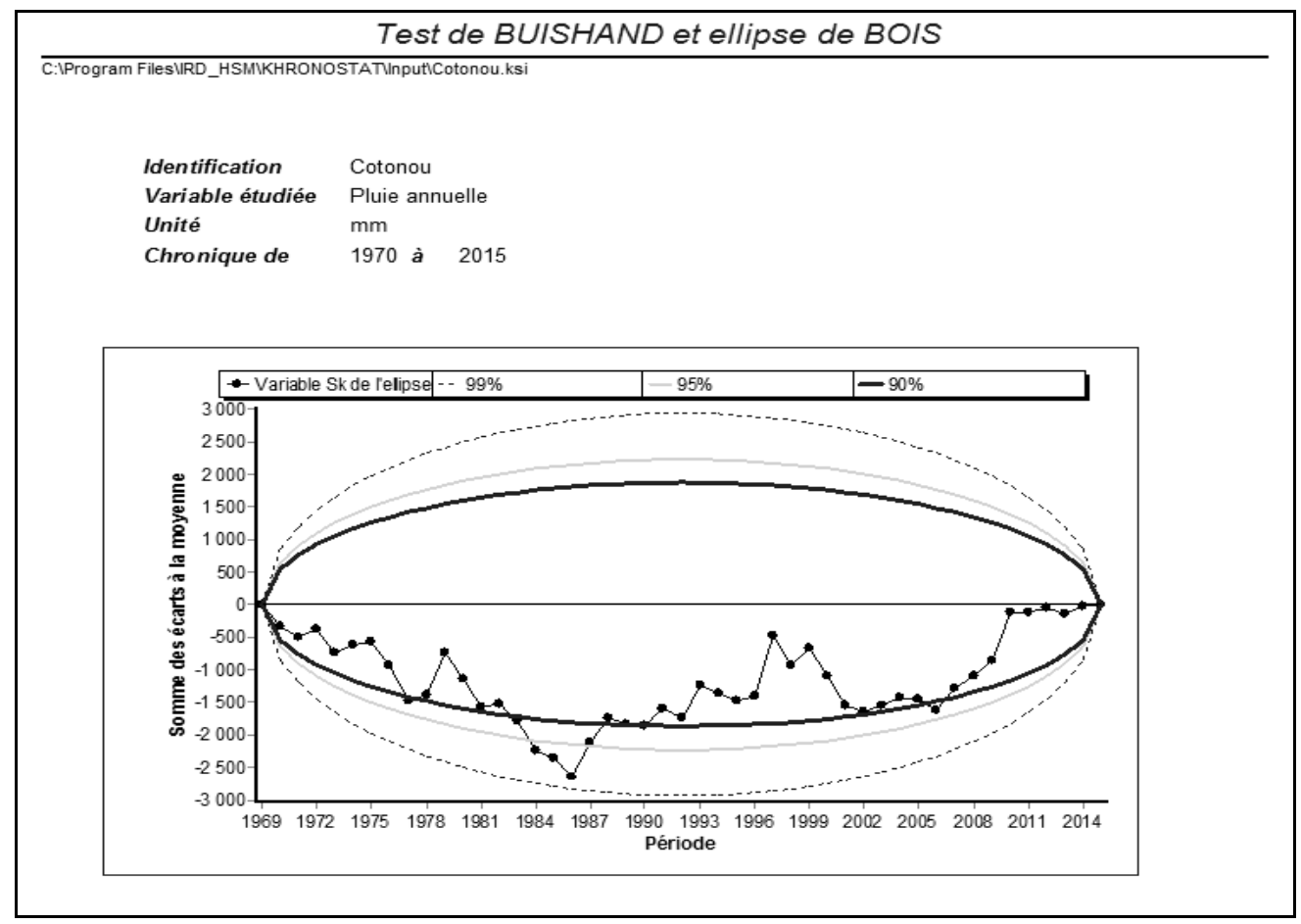

Figure 3 : Rupture de stationnarité par le test de Buishand à la station de Cotonou. 


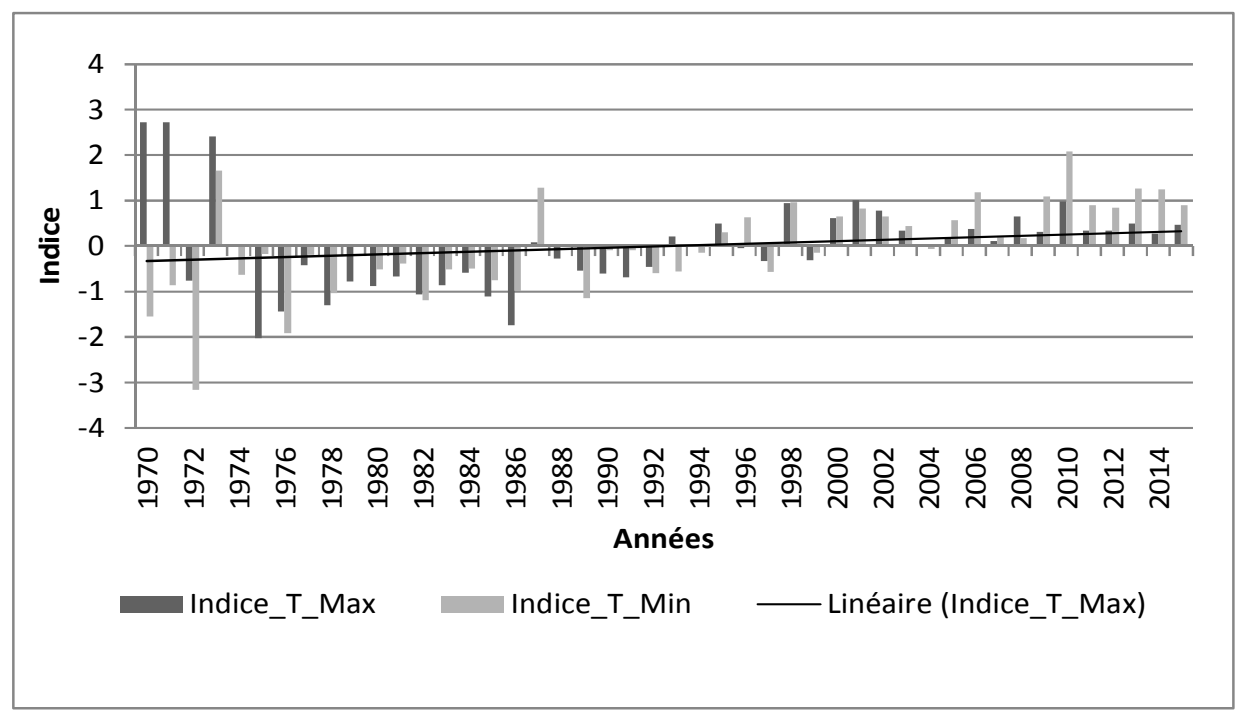

Figure 4 : Tendance thermométrique à Cotonou aéroport.

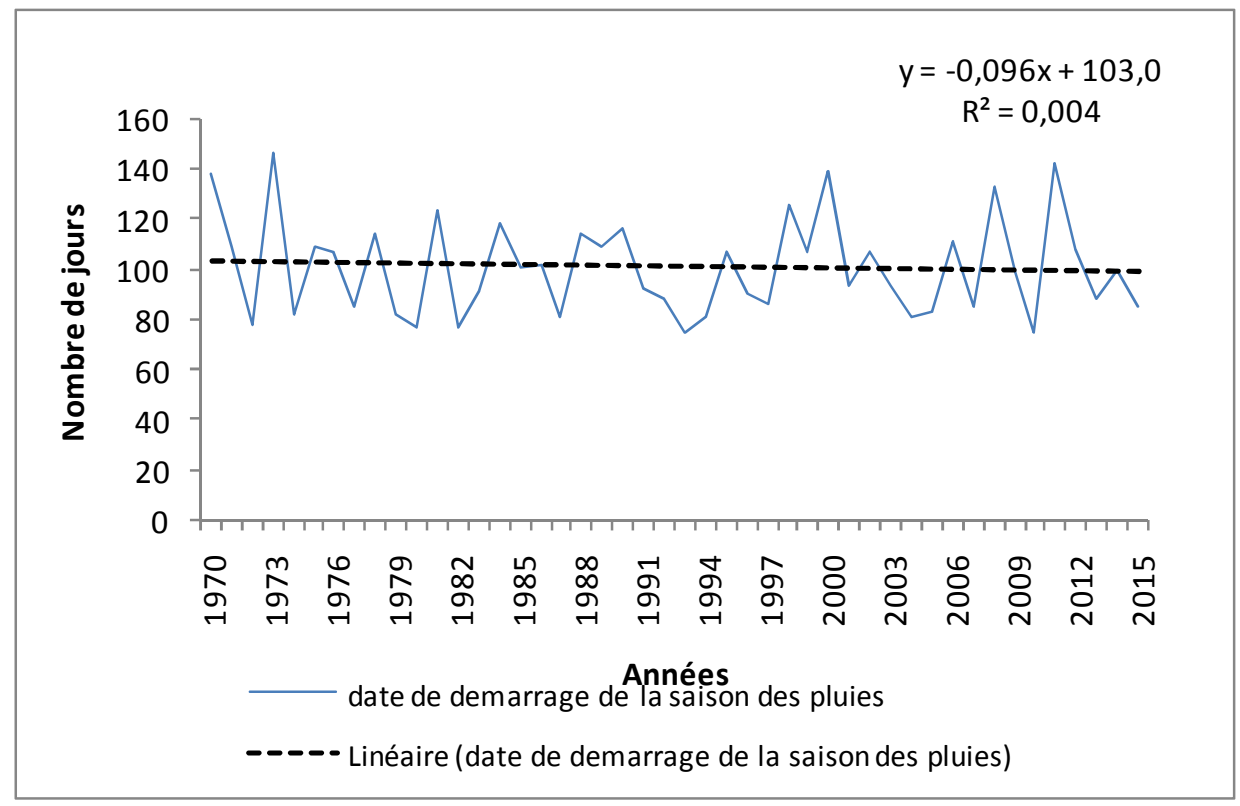

Figure 5 : Evolution du démarrage de la saison des pluies. 


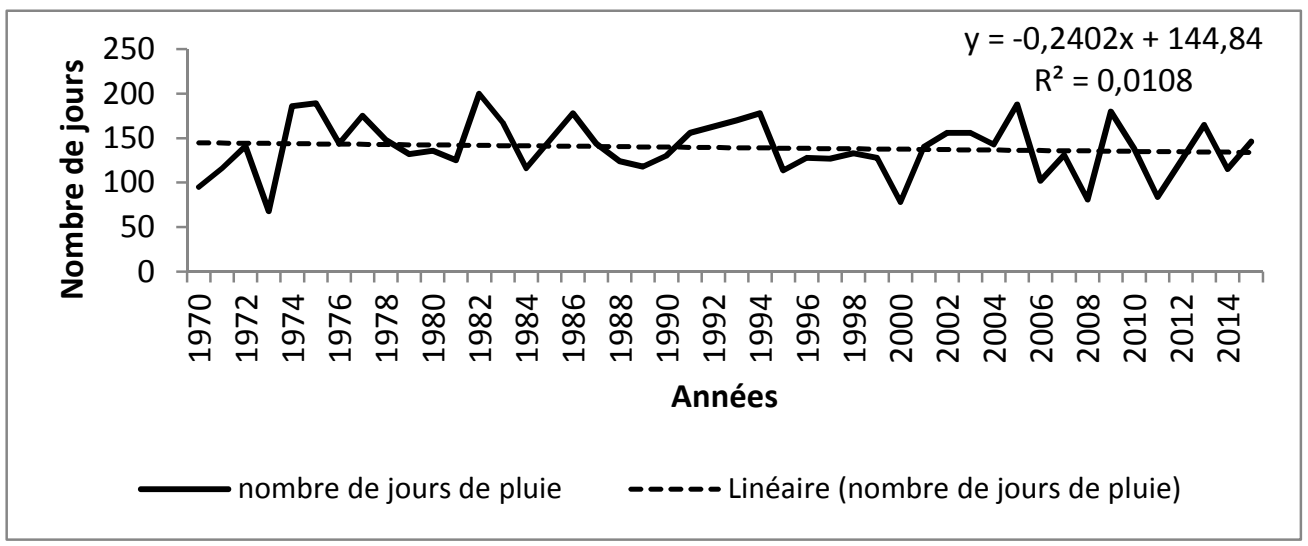

Figure 6 : Evolution interannuelle des longueurs des saisons pluvieuses entre 1970 et 2015.

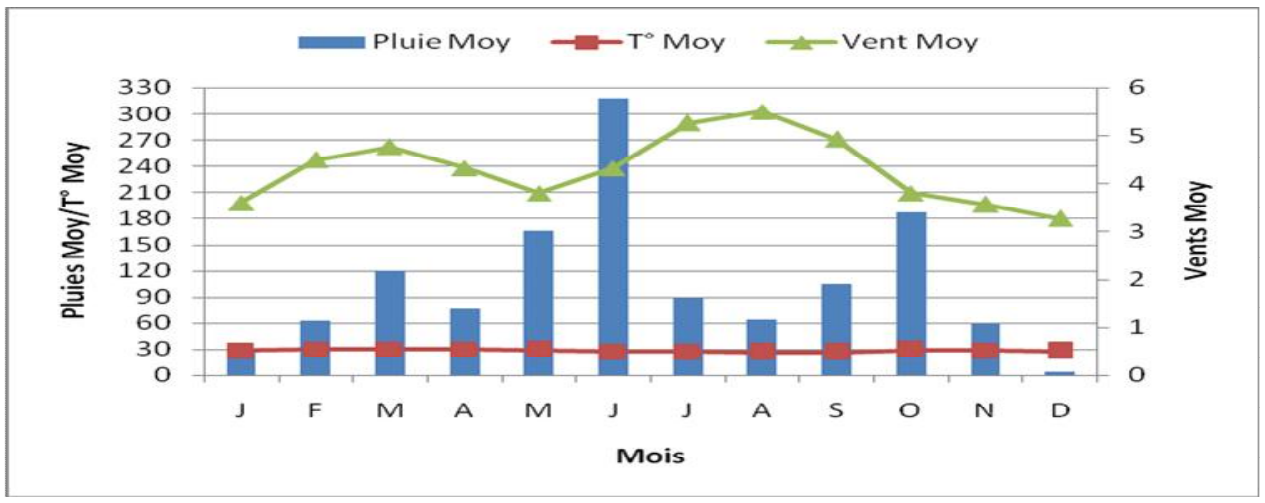

Figure 7 : Courbe ombro-thermique combinée à la courbe de la vitesse moyenne mensuelle du vent de la Commune de Sô-Ava 2014-2016.

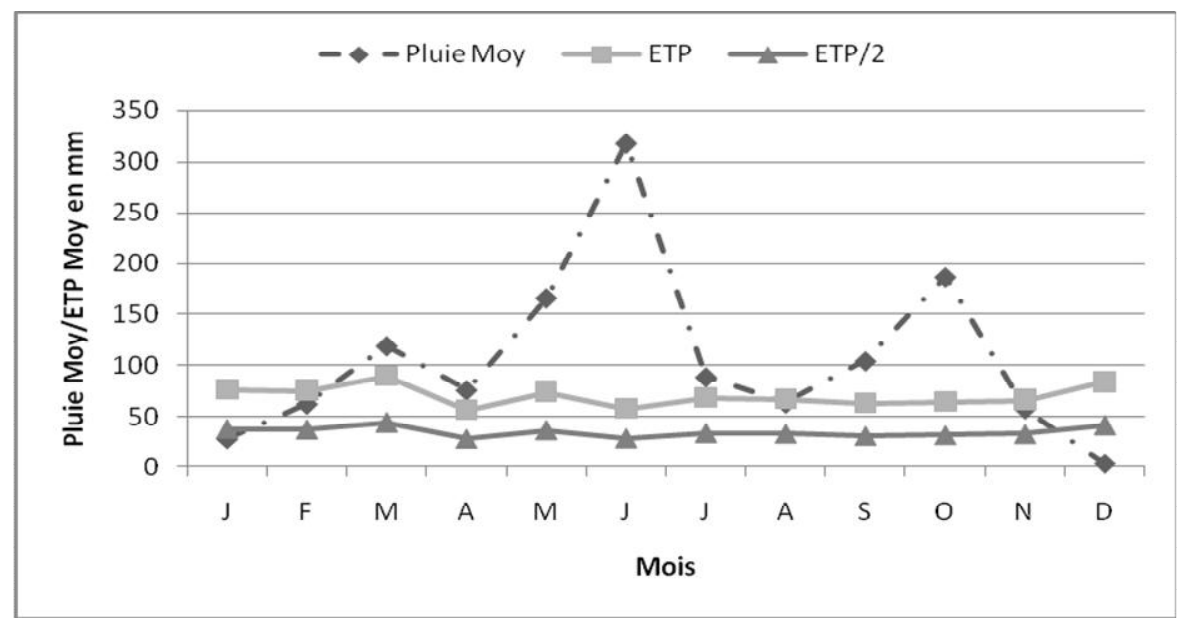

Figure 8 : Courbe de Franquin réalisée à partir des données climatiques recueillies de 2014 à 2016. 


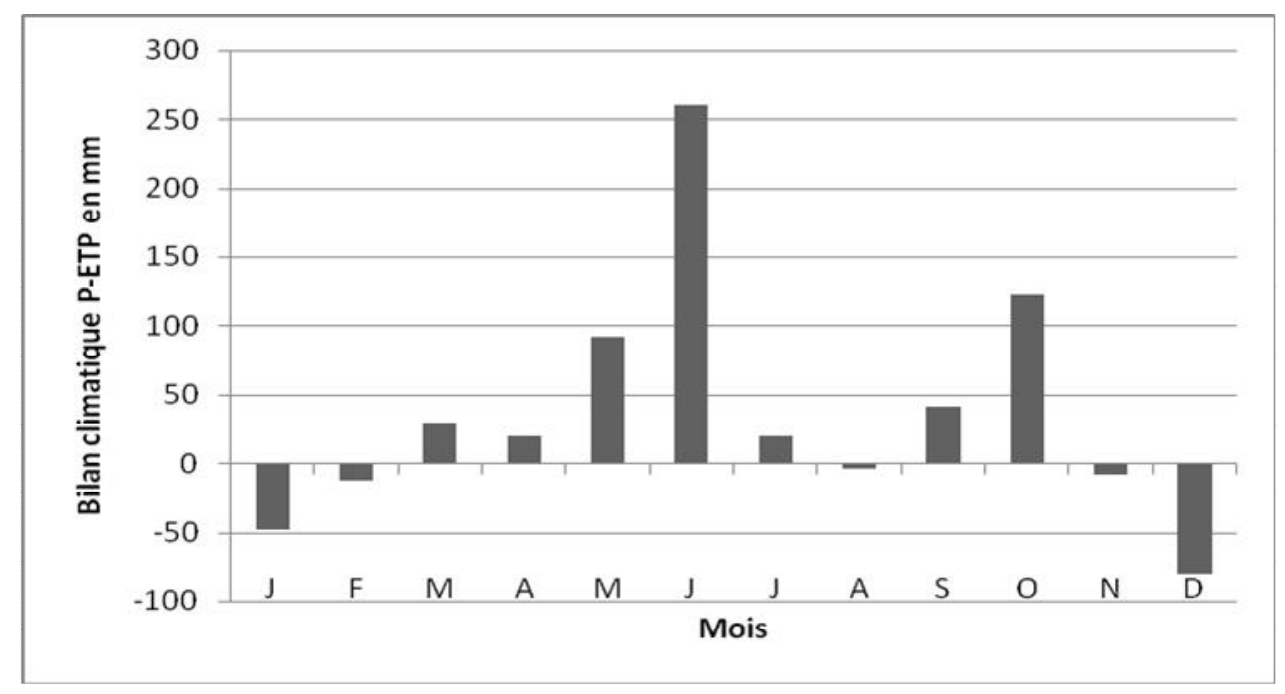

Figure 9 : Bilan climatique (P-ETP) sur la période 2014-2016.

Tableau 1 : Evolution des rendements moyens de la tomate, du piment et de la patate douce.

\begin{tabular}{lcccc} 
& $\mathbf{2 0 1 4}$ & $\mathbf{2 0 1 5}$ & $\mathbf{2 0 1 6}$ & $\begin{array}{c}\text { Taux de régression entre } \\
\text { 2014 et 2016 (\%) }\end{array}$ \\
\hline Tomate (t/ha) & 18,75 & 14,25 & 13 & $-0,31$ \\
\hline Piment (t/ha) & 6,5 & 5,75 & 4,3 & $-0,34$ \\
& & & & $-0,17$
\end{tabular}

\section{DISCUSSION}

Mauvaise couverture des besoins en eau des cultures dans la zone d'étude

D'après Servat et al. (1998), une rupture est définie comme un changement dans la loi de probabilité des variables aléatoires dont les réalisations successives définissent les séries chronologiques étudiées. L'existence de la modification brutale de certains paramètres statistiques notamment la moyenne est l'une des causes possibles de la rupture de l'homogénéité des séries chronologiques. Toutefois, les données obtenues et analysées par rapport à la production maraîchère dans la zone d'étude concernent le régime pluvial. Les résultats obtenus corroborent ceux de Pige et al. (2001) et Houssou (2014) selon qui la petite saison sèche tend à disparaître, laissant la place à une longue saison des pluies dont l'installation est plus tardive. Ceci a d'ailleurs été constaté au cours de la période d'étude (2014-2016) où tous les mois de 2014 ont été spécialement pluvieux. Cependant, en 2015, les mois de décembre et janvier n'ont reçu aucune pluie tandis qu'en 2016, ce sont plutôt, février et décembre qui n'en ont pas reçues. Cette variabilité pluviométrique observée dans la zone d'étude est conforme à ce qui a été observé dans la plupart des travaux sur le régime des précipitations de la sous-région de l'Afrique de l'Ouest (Servat et al., 1998 ; 
Noufe et al., 2011) et particulièrement au Bénin (Vissin, 2007; Vissin et al., 2007 ; Koumassi, 2014). Le Barbé et al. (2002) ont montré qu'au Niger, les vingt années (19711990) sont plus sèches que les vingt précédentes, et cela sans changement de la longueur de la saison des pluies.

Quand aux tendances thermométriques, le record d'anomalie de la température moyenne est supérieur à celui de $+0,55{ }^{\circ} \mathrm{C}$ calculé à l'échelle mondiale (IPCC, 2001) et $\mathrm{de}+1,1{ }^{\circ} \mathrm{C}$ au Cameroun (Sighomnou, 2004) sur la même année. Ce phénomène montre une certaine concordance des faits climatiques dans le temps et dans l'espace.

Toutefois, au cours de la période d'étude allant de 2014 à 2016, il faut signaler que toutes les saisons culturales ont été bonnes en matière de record pluviométrique au point où aucun déficit n'a été enregistré. Le hic, c'est l'abondance des pluies, leur mauvaise répartition, la réduction du nombre de jours et les vents à vitesse élevée avec des cultures souvent submergées d'eau et victimes de verses. Ces résultats confirment ceux obtenus par Ogouwalé (2006), Affo-Dogo (2012), Chede (2012), et Codjo (2016). En outre, ils confirment et complètent ceux de Houssou (2014). En clair, les analyses montrent combien de fois la dégradation des conditions pluviométriques dans la zone étudiée est hétérogène et impacte négativement le déroulement des saisons culturales.

\section{Bouleversement du calendrier agricole classique}

De nos jours, le calendrier agricole connaît un sérieux bouleversement du fait de l'imprévisibilité climatique (Houssou, 2014). Les producteurs n'arrivent plus à distinguer la séparation des saisons. La pluie tombe lorsqu'ils ne l'attendent pas et devient rare pendant la période où ils en ont le plus besoin. En effet, les meilleures dates de semis semblent aujourd'hui méconnues, suite aux irrégularités observées ces dernières années avec notamment le démarrage tardif et réel de la saison des pluies en avril plutôt qu'en mars comme autrefois. Mais le constat fait ces dernières années est que le mois de mars des trois dernières années a été pluvieux ainsi que celui de février des années 2014 et 2015. Certains producteurs, dans l'incertitude climatique avaient semé pour la grande saison en fin février- début mars avec la persistance des pluies. Mais très tôt, ils ont été rattrapés par des poches de sécheresse car souvent le volume des précipitations de février et mars était enregistré sur une moyenne de trois jours le mois. Les cultures sujettes au stress hydriques n'ont papas connu un bon développement et cela a constitué une perte pour ces producteurs qui ont dû reprendre le semis vers fin avril pour s'assurer une bonne productivité avec le réel démarrage de la saison des pluies. Codjo et al. (2013) ont affirmé que cette situation oblige parfois les producteurs à reprendre certaines opérations culturales telles que le labour, le semis, voire les traitements phytosanitaires et de ce fait, la main d'œuvre est doublement payée pour les mêmes opérations, augmentant ainsi la charge de production. D'un autre côté, les pesticides pulvérisés sur les feuilles des plantes sont fortement dilués, voire lavés. La prolifération des insectes ravageurs serait à craindre dans les champs de culture (FAO, 2007 ; GIEC, 2007). En effet, une hausse de la température cause aussi une prolifération des parasites, qui ne seront pas tués pendant l'hiver (phénomène du Winterkill) et qui pourront alors accomplir davantage de cycles reproductifs (Cammel et Knight, 1992, cités par Houssou, 2014). Cette situation exige de nouveaux traitements; ce qui augmente les charges de production au sein des exploitations. Enfin, les pluies abondantes, répétées et imprévisibles qui s'observent de plus en plus contribuent à des inondations précoces avant la crue de la rivière Sô qui de surcroît, vient détruire les cultures arrivant en fin de cycle en juillet voire août. Toutes ces conséquences ont pour corolaire le bouleversement du calendrier agricole, l'augmentation des charges de production et 
donc la diminution du revenu des maraîchers et pour y remédier les producteurs mettent en place différentes stratégies d'adaptation.

\section{Baisse du rendement des cultures}

Tous les producteurs enquêtés ont indiqué que la variabilité climatique contribue à la perte des récoltes et à la baisse des rendements des cultures. C'est une situation que viennent corroborer les résultats issus des essais conduits sur trois ans avec trois des principales cultures maraîchères que sont: la tomate, le piment et la patate douce. En effet, l'environnement de croissance et de développement des plantes est fortement perturbé ces dernières années et ne favorise plus une bonne production.

Tous les enquêtés sont unanimes sur le fait que la production maraichère, très dépendante de l'eau, est essentiellement marqué par de grandes variations saisonnières qui provoquent d'énormes dégâts sur les cultures et par ricochet entraînent une baisse drastique des rendements. Par exemple, les pluies de la grande saison ne sont pas efficaces et profitables aux cultures contrairement à celles de la petite saison car accompagnées de vents à fortes vitesses. L'évolution de la vitesse du vent a donc des conséquences sur les cultures maraîchères, se traduisant par l'accroissement des besoins en eau surtout en début de saison sèche ainsi qu'à la verse des cultures surtout dans le mois le plus ventilé (juillet). Par surcroît, les mois agricoles ont vu leurs températures moyennes augmenter ces dernières années. Cette hausse de la température augmenterait la cadence d'évaporation, réduisant ainsi le niveau d'humidité disponible pour la croissance des plantes (Atidégla, 2011). Il ressort de ces analyses que l'eau reste le facteur clé qui conditionne la production maraîchère. La situation agricole serait plus délicate dans les zones tropicales et on estime que globalement les rendements agricoles devraient baisser dans ces zones à cause de la fréquence des sécheresses et du stress hydrique. Selon les travaux de Ogouwalé (2006), les impacts des péjorations pluviométriques et de la hausse des températures sur les cultures se manifestent fréquemment par les stress hydriques et/ou thermiques. Le climat n'étant pas le seul facteur déterminant le rendement, les autres déterminants du rendement (fertilité du sol, qualité des semences, maladies et ravageurs...) participent aussi fortement à cette baisse des rendements perçue. Toutefois, d'après les résultats de plusieurs travaux menés dans des plaines et bas-fonds, Atidegla et al. (2017) affirment que les terres des plaines inondables sont plus productives que celles des plateaux car leur fertilité est renouvelée annuellement et de façon naturelle. Partant, dans la plaine d'Ahomey-Gblon, le problème d'infertilité des terres est à écarter. C'est d'ailleurs l'une des raisons pour laquelle Najlaoui (2010) a affirmé que l'option du choix variétal semble intéressant pour la durée de cycle de certaines cultures et permet de compenser l'augmentation de la température sur le raccourcissement de la phase de remplissage. Mais signalons aussi que de plus en plus les producteurs des plaines et bas-fonds sont souvent confrontés aux problèmes d'inondation qui leur créent parfois d'énormes pertes et influent négativement sur la production en régime pluvial du fait du non aménagement des sites avec système de maîtrise d'eau totale.

Pour le cas de la période d'étude, certaines parcelles ont été inondées même avant la survenance de la crue annuelle en Août; ce qui a engendré la submersion et la détérioration de plusieurs cultures dans la plaine.

Les travaux de Sossa (2001) ont cependant montré que du fait des variabilités climatiques, les modifications dans le bilan hydrique ne compromettent pas dangereusement le bouclage du cycle du maïs, la spéculation vivrière la plus cultivée dans le pays. Mais selon les mêmes auteurs, si le rythme des variations persiste, ce qui s'observe déjà, la production nationale du 
maïs sera hypothéquée. Toutefois, la garantie d'une bonne production est aussi tributaire des conditions liées à une bonne articulation des opérations culturales (défrichement, travail du sol, plantation ou semis, sarclage ou entretien, fertilisation ou non, usages ou non de variétés améliorées, etc.) (Noufe et al., 2015).

\section{Conclusion}

Les présents travaux ont révélé une forte variabilité pluvio-thermométrique, caractérisée par une succession des années déficitaires et des années excédentaires. L'évaluation des conséquences de ces perturbations pluviométriques sur la production agricole a également consisté à déterminer les dates de démarrage des pluies, leur raccourcissement et les dates leurs ruptures sur la période 1970-2016. L'analyse d'autres paramètres climatiques (Vents, ETP et Bilan climatique) sur la période 2014-2016 pour la production de la tomate, du piment et de la patate douce a permis d'expliquer les impacts de cette dégradation climatique dont notamment la baisse enregistrée par rapport aux rendements des trois cultures testées. Toutefois, les modifications des conditions climatiques ne suffisent pas à expliquer la dynamique d'ensemble du développement végétatif des cultures et ne saurait constituer un facteur limitatif majeur pour leur production. La connaissance du climat tient une place importante parmi les savoir-faire que développent les producteurs de la zone d'étude pour s'adapter aux contraintes du milieu et des sites de production. Toutefois, la stratégie favorable à eux pourrait être la pratique de l'agriculture de contre saison car présentant moins de risques par rapport au régime fluvial. Partant, des mesures d'accompagnement pouvant assurer la durabilité des activités dans la plaine inondable d'Ahomey-Gblon sont souhaitées. C'est pourquoi, des actions comme l'aménagement du site avec une maitrise totale de l'eau sont vivement attendues des autorités centrales et partenaires au développement.

\section{CONFLITS D'INTERETS}

Les auteurs déclarent qu'il n'y a aucun conflit d'intérêts.

\section{CONTRIBUTIONS DES AUTEURS}

SCA a procédé à l'enquête de terrain et à la rédaction de l'article. HDK s'est occupé du traitement statistique et ESH a procédé à la correction de tout le document.

\section{REFERENCES}

Affo-Dogo A. 2012. Vulnérabilité et stratégies d'adaptation des agriculteurs dans la région des plateaux au Togo face au changement climatique : cas de la communauté rurale de Kpimé. Mémoire de Master II, Centre Régional AGRYMETH, $\mathrm{p} 81$.

Atidegla CS, Koumassi HD, Mouzou ET, Houssou SE. 2017. Variabilité climatique et production du riz dans le bas-fond de Dokomey au Bénin. Actes du $1^{\text {er }}$ Colloque Scientifique International du Laboratoire Interface Sciences du Sol, Climat et Production Végétale de l'Université de Lomé, Togo, $\mathrm{p} 10$

Atidegla CS. 2012. Rapport d'étude des techniques d'aménagement des bas-fonds d'Allada et d'Abomey-Calavi au Bénin. Centre Régional pour la Promotion Agricole (CeRPA) Atlantique-Littoral, $\mathrm{p} 35$.

Atidegla CS. 2011. Effets des différentes doses d'engrais minéraux et de la fiente de volaille sur l'accumulation de biocontaminants et polluants (germes fécaux, composés azotés et phosphorés, métaux lourds) dans les eaux, les sols et les légumes de Grand-Popo au Bénin. Thèse de doctorat unique, EDP/FLASH, Université d'Abomey-Calavi (UAC), Bénin, p 319.

Balme DM. 2004. Analyse du régime pluviométrique sahélien dans une perspective hydrologique et agronomique. Etude de l'impact de sa variabilité sur la culture du mil. Thèse de doctorat. Institut National Polytechnique de Grenoble (France), p 163.

Buishand T. A. 1984. Tests for detecting a shift in the mean of hydrological time series. Journal of Hydrology, 58: 51- 69.

Chede F. 2012. Vulnérabilité et stratégies d'adaptation au changement climatique des 
paysans du Département des Collines au Bénin : cas de la Commune de Savè, Mémoire de Master II, Centre Régional AGRYMETH, p 86.

Codjo HT. 2016. Aménagement hydro-agricole pour la réduction de la vulnérabilité et l'adaptation de l'agriculture aux changements climatiques dans la commune d'Adjohoun. Mémoire de Master 2, Gestion des Risques Catastrophes. MIRD/FLASH, UAC, p 96.

Codjo HT, Lamodi F, Agbelessi S, Ogouwale R, Ogouwale E. 2013. Stratégies paysannes d'adaptation aux changements climatiques dans la Commune de Pobè', Actes du $26^{\text {ème }}$ Colloque de l'Association Internationale de Climatologie, Cotonou, 164-16.

Cocheme J, Franquin P. 1969. Une étude d'agro climatologie de l'Afrique sèche au Sud du Sahara en Afrique Occidentale, p 87.

Eldin M. 1989. Analyse et prise en compte des risques climatiques pour la production végétale, In : Le risque en agriculture, Dynamique des systèmes agraires', Orstom, collection à travers champs, Paris, 47 - 63 .

FAO 2007. Adaptation to climate change in agriculture, forestry and fisheries: perspectives, framework and priorities", Rome 2007, p 32.

Franklin P. 1968. Analyse agroclimatique en région tropicale: les conditions hydriques. s.1: Cahier ORSTOM, Série biologique, 5, 15-23.

GIEC 2007. Résumé à l'intention des décideurs, In : M.L. Parry, O.F. Canziani, J.P. Palutikof, P.J. van der Linden, et C.E. Hanson (Eds.), Bilan des changements climatiques: impacts, adaptation et vulnérabilité, Rapport $d u \quad$ Groupe d'Experts Intergouvernemental sur l'évolution du climat. Cambridge University Press, Cambridge, Royaume-Uni, p 114.

Houssou SE. 2014. Variabilité climatique et production maraîchère dans le bas-fond de Houinga-Houégbé (Commune de Houéyogbé). Mémoire de Master II, FSA/UAC, p 119.

INSAE (Institut National de la Statistique et de l'Analyse Economique) 2003. Troisième recensement général de la population et de l'habitation. Synthèse des résultats, p 34.
IPCC 2001. Incidences de l'évolution du climat dans les régions : Rapport Spécial sur l'Evaluation de la vulnérabilité en Afrique, Island Press, Washington, p 53.

Kouhoundji N. 2011. Problématique de la maîtrise de l'eau dans la vallée de l'Ouémé à Sô-Ava, Mémoire de Maîtrise en Géographie/ FLASH/UAC, p 65.

Koumassi DH. 2014. Risques hydro-climatiques et vulnérabilités des écosystèmes dans le bassin versant de la Sota à l'exutoire de Coubéri. Thèse de Doctorat Unique, EDP/FLASH, UAC, p 245.

Le Barbé L, Lebel T, Tapsoba D. 2002. Rainfall Variability in West Africa during the Years, 1950-1990. Journal of Climate, 15: 187-202.

Lubes-Niel H, Masson J-M, Serval E, Parture JE, Kouamé B. 1994. Caractérisation de fluctuations dans une série chronologique par l'application de tests statistiques, étude bibliographique. Programme ICCARE, ORSTOM, rapport n³, p 21 .

Mairie Abomey-Calavi 2012. Plan de développement communal, quinquennat 2012-2016, Abomey-Calavi, Bénin, p 125.

Martin C. 1986. Etude d'un agrosystème villageois dans la région du Zou (Bénin). Montpellier, Mémoire d'étude ENSSAA. $56 \mathrm{p}$.

MEPN 2008. Programme d'Action National d'Adaptation aux changements climatiques du Bénin (PANA- Bénin). Rapport de synthèse. Cotonou. $\mathrm{p} 81$.

Morel R. 2004. Le climat et l'implantation des hommes : le cas de la Côte d'Ivoire. Annales A IC, 1: 117-132.

Najlaoui H. 2010. Impact du changement climatique sur la production d'orge dans la zone de BEJA au Nord-Ouest de la Tunisie. European Scientific Journal, 11(9): 1-19.

Noufe D, Lidon B, Mahe G, Servat E, Brou YT, Koli BZ, Chaleard JL. 2011. Variabilité climatique et production de maïs en culture pluviale dans l'Est ivoirien. Hydrological Sciences Journal, 56(1) : 152 - 167.

Noufe D, Kouadio ZA, Soro GE, Wayou1 TP, Goula BTA, Savane I. 2015. Impact de la variabilité climatique sur la production du maïs et de l'igname en zones Centre et Nord de la Côte d'Ivoire. Agronomie Africaine, 27(3): $241-255$. 
Ogouwalé E. 2006. Changements climatiques dans le Bénin méridional et central : indicateurs, scénarios et prospective de la sécurité alimentaire. Thèse de Doctorat unique, LECREDE/ FLASH/ EDP/ UAC, p 302.

Pettitt AN. 1979. A Non-Parametric Approach to the Change-Point Problem. Journal of the Royal Statistical Society, 28(2): 126-135.

Pige J, Biaou F, Vigan O. 2001. Zonage géographique et typologie de fonctionnement des exploitations agricoles. Projet d'amélioration et de diversification des systèmes d'exploitation (PADSE) Départements de l'Alibori, du Borgou, des Collines et du Zou. Synthèse des résultats des missions d'appui au volet enquêtes villageoises (octobre 2000-mai 2001). PADSE, Parakou, Bénin, p 64.

PNUD 2007. Rapport mondial sur le développement humain 2007/2008 : Les objectifs du millénaire pour le développement : un pacte entre les pays pour vaincre la pauvreté humaine. Economica 49, rue Haricart, 75015 Paris, p 367.

Servat E, Paturel J, Kouame B, Travaglio M, Ouedraogo M, Boyer J, Lubes-Niel H, Fritsch J, Masson J, Marieu B. 1998. Identification, caractérisation et conséquences d'une variabilité hydrologique en Afrique de l'Ouest et Centrale. IAHS Publication, 323-338.

Sighomnou D. 2004. Analyse et redéfinition des régimes climatiques et hydrologiques du Cameroun: perspectives d'évolution des ressources en eau. Thèse de doctorat d'Etat es sciences naturelles, Yaoundé, Université de Yaoundé I, p 291.

Sossa B. 2001. Contribution à la mise en valeur de la plaine d'inondation du lac Toho à Kponou ( $\mathrm{S} / \mathrm{P}$ Athiémé, Département du Mono), Thèse d'Ingénieur Agronome, FSA/UNB, p 84.

UICN 2000. Union internationale pour la conservation de la nature et de ses ressources Groupe d'experts des plaines d'inondation sahéliennes. Vers une gestion durable des plaines d'inondation sahéliennes', UICN, Gland, Suisse et Cambridge, Royaume-Uni. $\mathrm{xii}+\mathrm{p} 214$.

Vissin E, Houndenou C, Perard J. 2007. Sécheresse pluviométrique et tarissement dans le bassin de la Mékrou (Bénin, Afrique de l'Ouest). Actes du $10^{\text {ème }}$ Colloque de AIC, Cathage Tunis ; 568-573.

Vissin EW. 2007. Impact de la variabilité et de la dynamique des états de surface du bassin versant du fleuve Niger. Thèse de doctorat, Université de Bourgogne, CRC, Dijon, p310. 